\title{
Dramatic response of BRAF V600E-mutant epithelioid glioblastoma to combination therapy with BRAF and MEK inhibitor: establishment and xenograft of a cell line to predict clinical efficacy
}

Yu Kanemaru', Manabu Natsumeda ${ }^{1 *}$ (D, Masayasu Okada ${ }^{1}$, Rie Saito², Daiki Kobayashi', Takeyoshi Eda', Jun Watanabe ${ }^{1}$, Shoji Saito ${ }^{1}$, Yoshihiro Tsukamoto ${ }^{1}$, Makoto Oishi ${ }^{1}$, Hirotake Saito ${ }^{3}$, Masayuki Nagahashi ${ }^{4}$, Takahiro Sasaki ${ }^{5}$, Rintaro Hashizume ${ }^{5}$, Hidefumi Aoyama ${ }^{3}$, Toshifumi Wakai ${ }^{4}$, Akiyoshi Kakita ${ }^{2}$ and Yukihiko Fujii ${ }^{1}$

\begin{abstract}
Epithelioid glioblastoma is a rare aggressive variant of glioblastoma (GBM) characterized by a dismal prognosis of about 6 months and frequent leptomeningeal dissemination. A recent study has revealed that $50 \%$ of epithelioid GBMs harbor three genetic alterations - BRAF V600E mutation, TERT promoter mutations, and homozygous deletions of CDKN2A/2B. Emerging evidence support the effectiveness of targeted therapies for brain tumors with BRAF V600E mutation. Here we describe a dramatic radiographical response to combined therapy with BRAF and MEK inhibitors in a patient with epithelioid GBM harboring BRAF V600E mutation, characterized by thick spinal dissemination. From relapsed tumor procured at autopsy, we established a cell line retaining the BRAF V600E mutation, TERT promoter mutation and CDKN2A/2B loss. Intracranial implantation of these cells into mice resulted in tumors closely resembling the original, characterized by epithelioid tumor cells and dissemination, and invasion into the perivascular spaces. We then confirmed the efficacy of treatment with BRAF and MEK inhibitor both in vitro and in vivo. Epithelioid GBM with BRAF V600E mutation can be considered a good treatment indication for precision medicine, and this patient-derived cell line should be useful for prediction of the tumor response and clarification of its biological characteristics.
\end{abstract}

Keywords: Epithelioid glioblastoma, BRAF V600E, Targeted therapy, Precision medicine

\section{Introduction}

Epithelioid glioblastoma is a rare aggressive variant of glioblastoma (GBM), newly proposed in the 2016 WHO classification, characterized by frequent leptomeningeal dissemination [1-5] and poor overall survival of approximately 6 months $[2,6]$. Genetic analyses have indicated a high percentage of BRAF V600E (50-93\%) [7, 8] and TERT promoter mutations (70\%), and homozygous deletions of $C D K N 2 A / 2 B$ (79\%) [7]. In a series of 14

\footnotetext{
* Correspondence: mnatsumeda@bri.niigata-u.ac.jp

${ }^{1}$ From the Departments of Neurosurgery, Niigata University, 1-757

Asahimachidori, Chuo-ku, Niigata, Japan

Full list of author information is available at the end of the article
}

epithelioid GBMs, 7 cases (50\%) harbored all 3 alterations [7], suggesting that epithelioid GBMs have recurrent genetic alterations, making them a prime candidate for precision-based medicine.

Progress in genetic studies and targeted therapies has vastly improved the treatment of some cancers $[9,10]$. Several case reports have described the success of targeted treatment to BRAF V600E-mutant brain tumors such as ganglioglioma [11, 12], pleomorphic xanthoastrocytoma (PXA) $[13,14]$ and papillary craniopharyngioma $[15,16]$. The VE-BASKET study investigating the efficacy of the BRAF inhibitor vemurafenib against $B R A F$ V600E-mutant cancers demonstrated that efficacy

(c) The Author(s). 2019 Open Access This article is distributed under the terms of the Creative Commons Attribution 4.0 International License (http://creativecommons.org/licenses/by/4.0/), which permits unrestricted use, distribution, and 
varied by histologic subtypes in patients with $B R A F$ V600E-mutant brain tumors [17], suggesting that some $B R A F$ V600E-mutant tumors have multiple concurrent drivers. It is still unclear which patients would benefit from precision medicine. Thorough genetic studies and establishment of patient-derived cell lines would support decision making in relation to targeted treatment.

Here we report a case of epithelioid GBM harboring $B R A F$ V600E mutation that showed a dramatic radiographical response to combined therapy with BRAF and MEK inhibitor after spinal dissemination. From a recurrent tumor sample obtained at autopsy, we established a cell line and used it to confirm both in vitro and in vivo that combination treatment was effective. Our findings suggest that targeted therapy would be beneficial for patients with epithelioid glioblastoma harboring $B R A F$ V600E mutation, and that establishment of cell lines and xenografts would be useful for predicting the effectiveness and overcoming the resistance mechanisms of precision-based treatments.

\section{Materials and methods Pathological analysis}

Informed consents for collection of samples during surgery and autopsy and their subsequent use for genetic analysis and other research purposes were obtained from the patient's family.

The surgical and autopsy specimens were fixed with $20 \%$ buffered formalin and embedded in paraffin. Histopathological examination was performed on 4- $\mu$ m-thick sections stained with hematoxylin and eosin (HE), and the KlüverBarrera method. The pathological diagnosis was made on the basis of the WHO classification of tumors of the central nervous system (CNS) by an experienced pathologist (AK). Immunohistochemistry (IHC) was performed as described previously using primary antibodies against Ki-67 (1:100, monoclonal, clone MIB-1, DAKO, Glostrup, Denmark), BRAF V600E (1:50, monoclonal, clone VE1, Spring Bioscience, Pleasanton, CA, USA) $[18,19]$ and phosphorylated ERK (pERK: 1:200, monoclonal, 9101, Cell Signaling Technology (CST), Danvers, MA, USA).

Surgically obtained brain tissue and orthotopic brain tumor tissue were also subjected to electron microscopy. Glutaraldehyde-fixed small tissue blocks were post-fixed with $1 \%$ osmium tetroxide, dehydrated through a graded ethanol series, and embedded in Epon 812. Ultrathin sections were then cut and stained with uranyl acetate and lead citrate, and examined with a Hitachi H-7100 electron microscope at $75 \mathrm{kV}$.

\section{Establishment of a BRAF V600E-mutant epithelioid glioblastoma cell line}

The NGT41 cell line was established from a disseminated lesion of the cervical spinal cord at autopsy (Fig. 1j), in accord with the protocol approved by the institutional review board at Niigata University (\#2016-2583). The specimen was minced with a scalpel and incubated in papain solution (Worthington Biochemical Corporation, Lakewood, NJ, USA) at $37^{\circ} \mathrm{C}$ for $30 \mathrm{~min}$ with shaking to dissociate the tissue. The tissue was then triturated using a sterile pipette. After centrifugation of the suspension, the cell pellets were maintained in Dulbecco's modified Eagle medium (DMEM) (Thermo Fisher Scientific, Waltham, MA, USA) supplemented with $10 \%$ fetal bovine serum (FBS) (Sigma Aldrich, St. Louis, MO, USA), 1\% AntibioticAntimycotic (Thermo Fisher Scientific) and $5 \mu \mathrm{g} / \mathrm{ml}$ Plasmocin (InvivoGen, Toulouse, France).

\section{Cell lines and drugs}

BRAF V600E-mutant glioblastoma cell lines, AM38 and DBTRG-05MG, were purchased from the Japanese Collection of Research Bioresources (JCRB) and the American Type Culture Collection (ATCC), respectively. Control $B R A F$-wildtype glioma cell lines, U87MG and T98G, were purchased from ATCC. All cell lines were grown in a humidified incubator at $37{ }^{\circ} \mathrm{C}$ under $5 \% \mathrm{CO}_{2}$. NGT41, AM38, U87MG, and T98G cells were grown as adherent monolayer cultures in 10\% FBS DMEM, and DBTRG05MG cells were grown in 10\% FBS RPMI1640 (Miltenyi Biotec, Aucurn, CA, USA) with Glutamax (Gibco, Paisley, UK). Dabrafenib (GSK2118436) was purchased from Selleck (Houston, TX, USA), and trametinib (GSK1120212) from AdooQ Bioscience (Irvine, CA, USA).

\section{Genetic characterization of tumor samples, cell lines and xenografts}

The CANCERPLEX cancer genome panel (KEW Inc., Cambridge, MA, USA) of 435 cancer-related genes (Additional file 1: Table S1) was used to assess the tumor sample, as described previously [20]. DNA was extracted from fresh frozen tissue using the QIAamp Blood \& Tissue Kit (Qiagen, Valencia, CA, USA) and from FFPE tumor tissue using the QIAmap DNA FFPE Tissue Kit (Qiagen). Direct sequencing of BRAF V600E and the TERT promoter was performed as reported previously [21]. Twenty nanograms of complimentary DNA was amplified using primers for the detection of $B R A F$ V600E and the TERT promoter. The primer sequences were: BRAF V600E; forward 5'-TCATAATGCTTGCT CTGATAGGA-3' and reverse 5'-GGCCAAAAATTT AATCAGTGGA-3', TERT promoter; forward 5'-GTCC TGCCCCTTCACCTT-3' and reverse 5'-CAGCGCTG CCTGAAACTC-3'. The PCR products were then sequenced on a 3130xl Genetic Analyzer (Applied Biosystems, Foster City, CA, USA) with a Big Dye Terminator v1.1 Cycle Sequencing Kit (Applied Biosystems) in accordance with the manufacturer's instructions. Multiplex ligation-dependent probe amplification (MLPA) was 

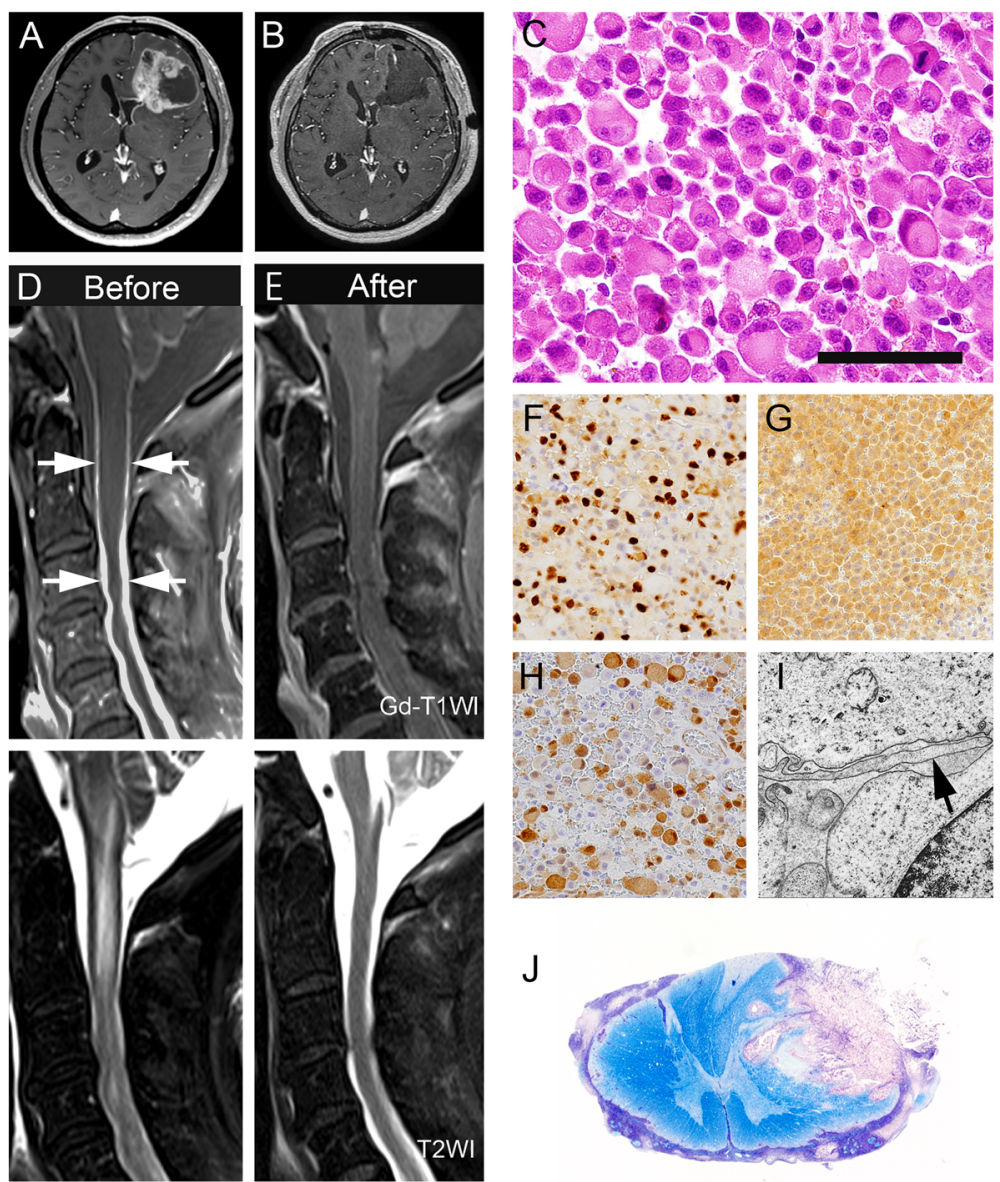

Fig. 1 Clinicopathologic features of the patient. Preoperative (a) and postoperative (b) brain MR images of the lesion. Spinal cord MR images before (d) and after (e) combination therapy with BRAF and MEK inhibitor. Histopathologic features of the surgical (c, $\mathbf{f}, \mathbf{g}, \mathbf{h}, \mathbf{i}$; frontal lobe) and autopsy (j; second segment of the cervical cord) samples. T1-weighted gadolinium-enhanced image demonstrating enhancement of the well circumscribed tumor in the left frontal lobe (a) and confirming the subtotal resection except for enhancement of the lateral ventricle (b). Before targeted treatment, thick leptomeningeal dissemination (arrow) and syringomyelia are evident in the upper cervical cord on post-contrast MR images and T2-weighted images (d). After 4 weeks of treatment, T1-weighted gadolinium-enhanced images and T2-weighted images (e) reveal a dramatical radiological response to the therapy. The tumor cells are composed of discohesive, rounded cells with rhabdoid morphology, showing brisk mitotic activity (c). MIB-1 labeling index 36\% (f). Positive immunoreactivity for BRAF V600E (g) and nuclear positivity for pERK (h).

Ultrastructure of the tumor cells. A few foot processes (arrow) are not interwoven (i). Spinal cord invasion by the tumor cells with leptomeningeal dissemination (j). Scale bars c: $20 \mu \mathrm{m} ; \mathbf{f}-\mathbf{h}: 150 \mu \mathrm{m}, \mathbf{i}: 2 \mu \mathrm{m}, \mathbf{j}: 500 \mu \mathrm{m}$

selected to assess the $C D K N 2 A / 2 B$ copy number. The SALSA MLPA probe mix P088-C2 (MARC-Holland, Amsterdam, the Netherlands) was used. In accordance with the manufacturer's instructions, $250 \mathrm{ng}$ of the DNA was denatured, hybridized, ligated, and subjected to PCR. The carboxyfluorescein (FAM)-labeled MLPA PCR products were separated by capillary electrophoresis on an ABI 3730xl DNA Analyzer (Applied Biosystems). The fragment lengths of the data were analyzed using Peak Scanner v2.0 (Thermo Fisher Scientific). Probe ratios below 0.4 were regarded as homozygous, and those from 0.4 to 0.7 were regarded as heterozygous deletions [22].

Droplet digital polymerase chain reaction (ddPCR) reagents and Primer/probe mix for BRAF V600E were purchased from Bio-Rad (Hercules, CA, USA). A $20-\mu \mathrm{L}$ aliquot of PCR mix, composed of $10 \mu \mathrm{L}$ of $2 \times$ ddPCR Supermix for Probes (No dUTP) (Bio-Rad), $1 \mu \mathrm{L}$ ddPCR Mutation Assay (Bio-Rad), and $9 \mu \mathrm{L}$ tumor DNA was loaded into each sample well of an 8-channel disposable droplet generator cartridge (Bio-Rad). An additional $70 \mu \mathrm{L}$ of droplet generation oil (Bio-Rad) was loaded into the oil well for each channel. After droplet generation, droplets were transferred to a 96-well PCR plate and subjected to thermal cycling. Amplification of the $20 \mu \mathrm{L}$ reaction mixture was carried out on a QX200 Droplet digital PCR system (Bio-Rad). After PCR, the 96-well PCR plate was transferred to a QX-200 droplet reader (Bio-Rad), and the data were analyzed using QuantaSoft analysis software (Bio-Rad). BRAF V600E mutation-specific signals were generated in the FAM channel, 
whereas BRAF V600E wildtype signals were generated in the HEX channel.

\section{Cell viability}

Fifteen hundred cells per $100 \mu \mathrm{L}$ medium were seeded into 96-well plates for one day and $10 \mu \mathrm{L}$ of each drug was applied for $72 \mathrm{~h}$. As a background control ( $0 \%$ control), $0.2 \%$ Triton X-100 was added to the control wells. To each well was then added $10 \mu \mathrm{L}$ of WST-8 (Nakalai Tesque, Kyoto, Japan), and the cells were incubated for $4 \mathrm{~h}$. Absorbance at $450 \mathrm{~nm}$ was measured in each well using ELx808 (BioTek Instruments, Winooski, VT, USA). Cell viability was quantified as: cell viability $(\%)=($ Asample $-\mathrm{a} 0 \%) /(\mathrm{a} 100 \%-\mathrm{a} 0 \%) \times 100$, where Asample is the sample absorbance, $\mathrm{a} 0 \%$ is the average $0 \%$ control absorbance, and a $100 \%$ is the average $100 \%$ control absorbance.

The normalized growth rate inhibition (GR) analysis was performed to minimize cell line-dependent differences in rate of cell division [23]. Fifteen hundred cells per $100 \mu \mathrm{L}$ medium were seeded into 96-well plates for one day and cell viability was measured at the time of treatment, and at 48 and $72 \mathrm{~h}$ after each drug administration using WST-8. Dabrafenib was diluted ranging from $1 \mathrm{nM}$ to $1000 \mathrm{nM}$ and trametinib from $0.1 \mathrm{nM}$ to $100 \mathrm{nM}$ in 7 increments. $0.5 \%$ DMSO was added to the control well. GR metrics and the $\mathrm{GR}_{50}$ was calculated using the Online GR Calculator (www.grcalculator.org/ grcalculator).

\section{Apoptosis assay and cell cycle analysis}

Apoptosis and the cell cycle were assessed using a Muse $^{\mathrm{Tm}}$ Cell Analyzer (Millipore, Billerica, MA, USA) with Annexin V and Dead Cell Assay and Cell Cycle analysis kits following the manufacturer's instructions, as described previously [24]. In brief, after the indicated treatments (dabrafenib; $10 \mathrm{nM}$, trametinib; $1 \mathrm{nM}$, combined; dabrafenib $10 \mathrm{nM}+$ trametinib $1 \mathrm{nM}$ ) for $48 \mathrm{~h}$, the cells were harvested, centrifuged at $300 \times \mathrm{g}$ for $5 \mathrm{~min}$, and washed once with $1 \times$ PBS. For the apoptosis assays, a $100-\mu \mathrm{L}$ cell suspension was labeled with Annexin V and Dead Cell Reagent (7-Amino-Actinomicyn D (7-AAD)) for $20 \mathrm{~min}$ at room temperature in the dark, and then analyzed. For cell cycle analysis, the cells were fixed in $70 \%$ ethanol for at least $3 \mathrm{~h}$, then washed with PBS and stained with $200 \mu \mathrm{L}$ propidium iodide (PI) for $30 \mathrm{~min}$. After staining, the cells were processed for cell cycle analysis.

\section{Western blotting}

Proteins were extracted using cell lysis buffer, and Western blotting was performed as described previously [25]. The blotted membranes were probed with anti-MEK (1: 500, CST), anti-pMEK (1:500, CST), anti-ERK (1:1000,
CST), anti-pERK (1:500, CST) and anti- $\beta$-actin (1:000, CST), and the signals were detected with an ECL system (Bio-Rad, Hercules, CA, USA).

\section{Subcutaneous and intracranial xenografts}

Four-to-five-week-old male BALB-c nu/nu mice were purchased from Charles River Laboratories Inc. (Yokohama, Japan) and housed under aseptic conditions, which included filtered air and sterilized food, water, bedding, and cages. The mice were anesthetized with medetomidine hydrochloride $(0.3 \mathrm{mg} / \mathrm{kg})$, midazolam $(4.0 \mathrm{mg} / \mathrm{kg})$ and butorphanol tartrate $(5.0$ $\mathrm{mg} / \mathrm{kg}$ ) by intraperitoneal injection.

For the subcutaneous tumor model, $1 \times 10^{6}$ NGT41 cells were suspended in $50 \mu \mathrm{L}$ of Neurobasal Medium (Gibco) and injected subcutaneously into the hip. To investigate the efficacy of treatment, these mice received vehicle control (0.5\% hydroxypropyl methylcellulose plus $0.2 \%$ Tween 80 , and 20\% dimethyl-sulfoxide (DMSO)) or combined treatment $(20 \mathrm{mg} / \mathrm{kg}$ dabrafenib and $2 \mathrm{mg} /$ $\mathrm{kg}$ trametinib solubilized in $20 \%$ DMSO) by oral gavage for 10 days. The tumors were measured daily with calipers, and tumor volume was calculated using the formula: Tumor volume $\left(\mathrm{mm}^{3}\right)=$ Length $(\mathrm{mm}) \times$ Width $(\mathrm{mm})^{2} / 2$ [26]. Two additional mice from each treatment group were used for histopathological and Western blot analyses as described above. These mice were sacrificed at $2 \mathrm{~h}$ after completion of treatment on the fifth day in order to collect tumor tissues.

For the intracranial xenograft model, a NGT41 cell suspension $\left(1 \times 10^{5}\right.$ cells in $2 \mu \mathrm{L}$ of Neurobasal Medium) was stereotactically injected into the right caudate putamen at $1.0 \mathrm{~mm}$ to the right of the midline, just behind the bregma, and at $3.0 \mathrm{~mm}$ depth. The mice were monitored daily and euthanized if body weight loss exceeded $20 \%$, or if they developed neurological symptoms indicative of tumor burden. Brain tissue was subsequently resected and subjected to formalin fixation and paraffin embedding.

All animal studies were approved by the Animal Use and Care Committee of Niigata University, and all of the animals were cared for and treated humanely in accordance with the Institutional Guidelines for Experiments Using Animals.

\section{Statistical analysis}

Differences between three or more groups were assessed using two-way analysis of variance (ANOVA) with the $p$-value threshold adjusted by Bonferroni's correction (i.e. $p<0.025$ for 3 groups, $p<0.0125$ for 4 groups) unless otherwise specified. Error bars represent standard error of the mean. Kaplan-Meier survival curves for intracranial xenografts were assessed by Log-rank test, and $p<0.05$ was considered statistically significant 
unless otherwise specified. All statistical tests were performed using the GraphPad Prism 6 software package (GraphPad Software, La Jolla, CA, USA).

\section{Results}

\section{Case report}

A 57-year-old man presented with headaches and slight dysphasia. Post-contrast MR images revealed a well circumscribed mass lesion measuring $6 \mathrm{~cm} \times 5 \mathrm{~cm}$ at the left frontal lobe, adjacent to the lateral ventricle. Subependymal enhancement was noted inside the left lateral ventricle (Fig. 1a). The intraparenchymal enhancing tumor was subtotally removed; the portion of the mass surrounding the lateral ventricle was left in situ (Fig. 1b). Examination of HE sections showed highly cellular, discohesive and medium-sized rounded cells with abundant eosinophilic cytoplasm and laterally positioned nuclei (Fig. 1c). Microvascular proliferation was rare; however, prominent necrosis was observed. The MIB-1 labeling index was about 36\% (Fig. 1f). Immunohistochemistry showed that the tumor cells were positive for BRAF V600E (Fig. 1g) and pERK (Fig. 1h). Ultrastructurally, the foot processes were not interwoven and lacked the macula adherens, unlike the common appearance in glioblastoma (Fig. 1i). The pathological diagnosis was epithelioid GBM. After surgery, the patient received intensity-modulated radiation therapy and concomitant temozolomide. During radiation, the patient became comatose and post-contrast brain MR images revealed dissemination and hydrocephalus. External ventricular drainage was performed to control the hydrocephalus and irradiation was continued, enlarging the radiation field to include the whole brain. After completion of concomitant treatment, a ventriculo-peritoneal shunt was performed, but the patient subsequently developed paraplegia. Spinal MR images revealed thick spinal dissemination and syringomyelia (Fig. 1d).

$B R A F$ V600E and TERT promoter (C250T) mutations, and $C D K N 2 A / 2 B$ loss were identified using the CANCERPLEX comprehensive genomic panel. No other genetic mutations were found. These genetic changes were confirmed by Sanger sequencing and MLPA analysis (Additional file 2: Figure S1). The fractional abundance (FA) of BRAF V600E calculated by ddPCR was $52.1 \%$ (Additional file 2: Figure S2). Although whole spinal irradiation was commenced, the patient's neurological conditions clearly worsened and a decline in the level of consciousness and dysphagia were observed, thus irradiation was stopped at 15 Gy (5 fractions). Following accelerated IRB approval for off-label use and written informed consent, the patient received $150 \mathrm{mg}$ of dabrafenib twice a day and $2 \mathrm{mg}$ of trametinib daily. A total of 4 weeks of targeted treatment was administered, alternating with 5 more fractions of whole spinal radiation to a total dose of $30 \mathrm{~Gy}$. After 4 weeks of treatment, spinal MR images demonstrated almost complete disappearance of the dissemination and syringomyelia (Fig. 1e). Unfortunately, the patient's paraplegia did not improve and he was taken off targeted treatment, mainly due to lack of funding. The patient was subsequently treated with temozolomide and bevacizumab, but succumbed to the disease 8 months after surgery.

At autopsy, macroscopic examination of the spinal cord revealed a thickened and whitish subarachnoid membrane, and the subarachnoid space was largely filled with tumor cells showing invasion into the dorsal part of the second segment of the cervical cord (Fig. 1j). A few remaining tumor cells formed small perivascular sheets within and around the surgically treated lesion in the left frontal lobe. However, no tumor cells were detectable in other parenchymal areas and visceral organs. Morphologically, the tumor cells shared features similar to those in the surgical sample, except for the mucinous component at autopsy.

\section{Characterization of NGT41 cells and xenografts}

NGT41 cells displayed robust growth and sustainable propagation in adherent cell culture. The NGT41 cell line retained the BRAF V600E and TERT promoter (C250T) mutation, and heterozygous deletion of $C D K N 2 A / 2 B$ (probe ratios were between 0.4 and 0.7), observed in the tissues obtained at surgery and autopsy (Additional file 2: Figure S1A, B).

The intracranial xenograft tumors formed well circumscribed masses in the brain, and showed dissemination into the subarachnoid with perivascular infiltration (Fig. 2a), morphologically resembling epithelioid cells with a mucinous component (Fig. 2b). These features closely resembled the patient's tumor at autopsy (Fig. 2d, e). Moreover, ultrastructural examination of the intracranial xenograft tumors revealed rounded tumor cells characterized by few foot processes and rich cytoplasmic organelles (Fig. 2c), similar to the tissue obtained at surgery (Fig. 2f).

\section{In vitro studies}

We evaluated the sensitivity of NGT41 and other BRAF V600E-mutant cell lines to treatment with dabrafenib and/or trametinib. The cell lines were treated with either dabrafenib (at $0,1,10,100$, or $1000 \mathrm{nM}$ ) or trametinib (at $0,0.1,1,10$, or $100 \mathrm{nM}$ ), or with both (combined; dabrafenib $+1 / 10$ trametinib) for $72 \mathrm{~h}$. All BRAF V600Emutant cell lines were sensitive to both drugs. In NGT41, addition of trametinib did not significantly reduce cell viability compared to dabrafenib alone. Among $B R A F$ V600E wildtype cell lines, U87MG showed a response to trametinib, consistent with a previous report (Fig. 3a) [27]. Next, we calculated growth rate inhibition 


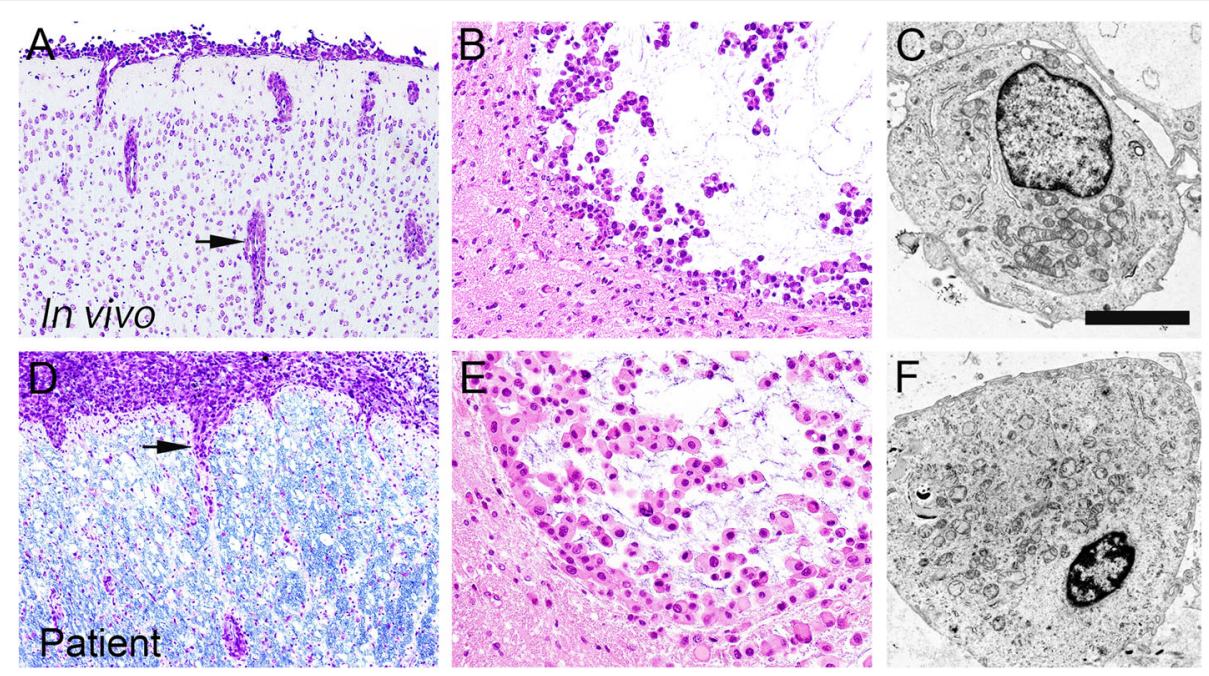

Fig. 2 Morphological similarity of the tumor cells between those in the orthotopic xenograft $(\mathbf{a}, \mathbf{b}, \mathbf{c})$ and those from the patient $(\mathbf{d}, \mathbf{e}, \mathbf{f})$. The orthotopic xenografts share strikingly similar characteristics to those of the autopsy specimen, including the mode of tumor infiltration (a, $\mathbf{d}$ ) and the light microscopic (b,e) and ultrastructural (c, f) morphology of the tumor cells. Scale bars a, d: $200 \mu \mathrm{m} ; \mathbf{b}$, e: $50 \mu \mathrm{m} ; \mathbf{c}, \mathrm{F}: 2 \mu \mathrm{m}$

in NGT41 and U87MG to account for possible cell linedependent differences in rate of cell division. Indeed, combination treatment in NGT41 showed a dramatic reduction in growth rate, whereas only a minimal reduction in growth rate was observed after combined treatment in U87MG (Additional file 2: Figure S3).

To assess the effects of dabrafenib and trametinib on the MAPK signaling pathway, we used Western blot analysis to examine the protein expression of key pathway components. Cells were treated with the blank control (0.5\% DMSO) or each drug (10 nM dabrafenib or 1 $\mathrm{nM}$ trametinib or combined; $10 \mathrm{nM}$ dabrafenib $+1 \mathrm{nM}$ trametinib) for $1 \mathrm{~h}$. Treatment with dabrafenib reduced the expression of both pMEK and pERK in BRAF V600E-mutant cell lines. Treatment with trametinib reduced the expression of pERK regardless of BRAF V600E status (Fig. 3b). In NGT41, combined treatment led to profound decreases in pMEK and pERK.

$B R A F$ V600E-mutant cell lines, but not BRAF wildtype cell lines, treated with dabrafenib and trametinib in combination exhibited significantly greater apoptosis (Additional file 2: Figure S4A; one-way ANOVA with Bonferroni's correction) and G0/G1 arrest (Additional file 2: Figure S4B).

\section{In vivo studies}

As the combination of BRAF and MEK inhibitors showed increased efficacy against NGT41 in vitro, we next tested the antitumor activity of this combination therapy using orthotopic human tumor xenografts. We transplanted NGT41 subcutaneously into the hip of BALB-c nu/nu mice, and treated the mice with control vehicle or dabrafenib and trametinib in combination daily by oral administration from day 5 of implantation for 10 days. Combined treatment significantly suppressed tumor growth in vivo compared with the vehicle control $(79.45 \pm 8.27$ (mean \pm SEM) vs $61.24 \pm 9.36 p<$ 0.0021 at day 5 , and $p<0.0001$ at days $6-11$ after initiation of treatment) (Fig. 4a). We removed the tumors from the mice after completion of treatment on day 5 . Histopathological examination showed that combined treatment markedly decreased the numbers of viable tumor cells (Fig. 4b). Western blot analysis showed that combined treatment suppressed the expression of pMEK and pERK in vivo (Fig. 4c).

We then sought to prove the efficacy of combination treatment on intracranial tumor models. We transplanted NGT41 cells into the mouse brain by stereotactic injection and treated the mice with vehicle control or combined treatment for 14 days. Combination treatment resulted in only a modest improvement in survival $(p=$ 0.0299, Log Rank test) (Fig. 4d). Western blot analysis of the tumor in an intracranial xenograft mouse treated with the combined treatment for 3 days before sacrificing showed marked reduction of pMEK and pERK after treatment compared to control (Additional file 2: Figure S5A). Also, serial body weight measurements were unchanged in the combination treatment group compared to control (Additional file 2: Figure S5B). HE sections of brain tumors in the treatment group at endpoint showed tumors of the same high-cellularity as the control group (Additional file 2: Figure S5C).

\section{Discussion}

Recent phase 3 clinical trials have advocated the usefulness of a combination of the BRAF inhibitor dabrafenib 


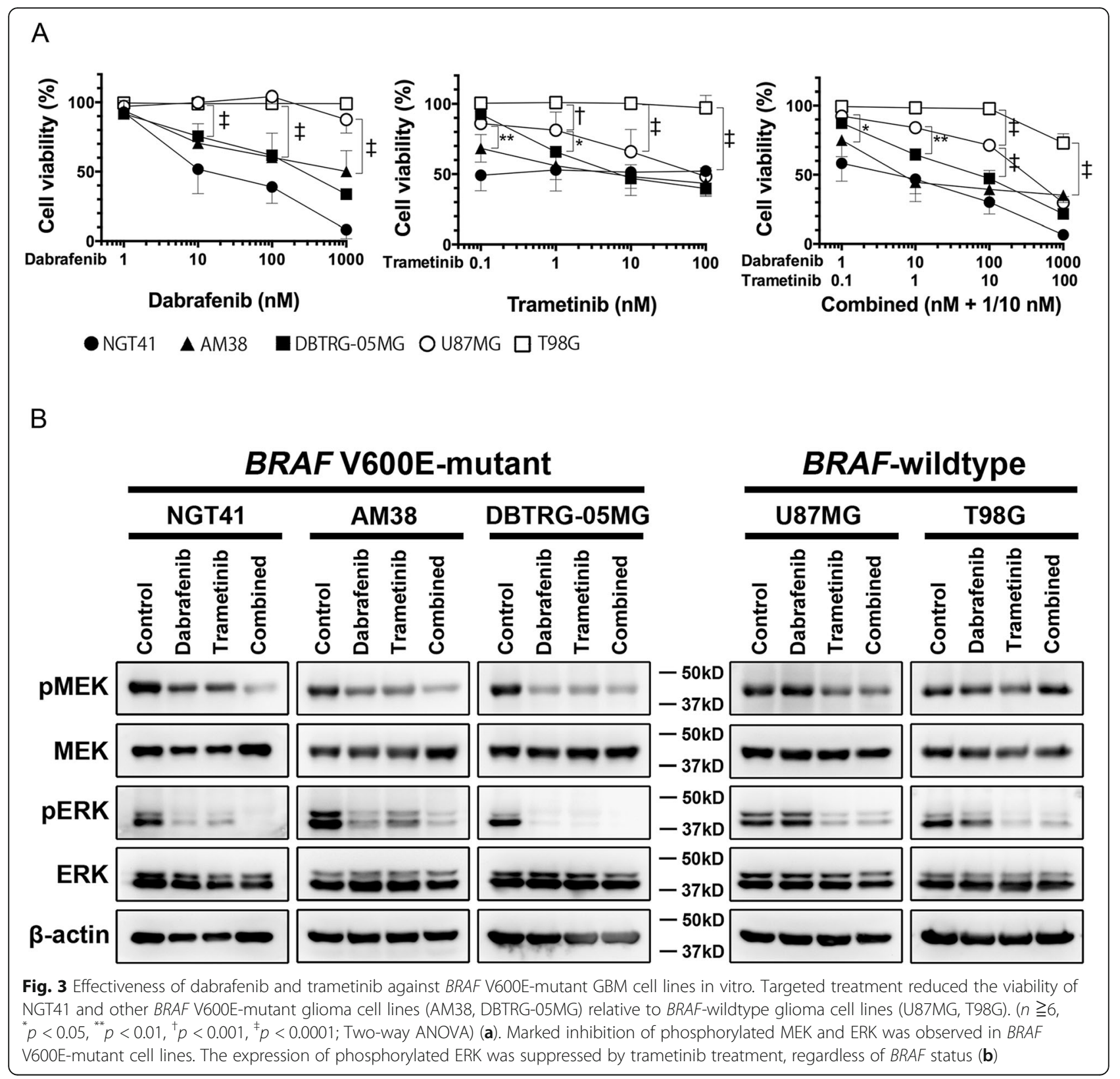

and the MEK inhibitor trametinib as first-line treatment for BRAF V600E-mutant metastatic melanomas [28, 29], as BRAF inhibitors alone cause resistance by reactivation of the MAPK pathway due to PDGFRB upregulation or NRAS mutation [30]. A preclinical study has demonstrated the efficacy of a combination of the BRAF inhibitor PLX4720 and the MEK inhibitor PD0325901 against BRAF V600E-mutant GBM [31]. The present in vitro and in vivo results were generally in agreement with those previous data.

The VE-BASKET study showed that vemurafenib alone was effective in only $9.1 \%$ of malignant diffuse gliomas, compared to $42.9 \%$ of PXAs [17]. This suggests that in malignant gliomas harboring BRAF V600E mutations, other pathways such as PI3K-Akt-mTOR pathway are also activated, $B R A F$ V600E mutations are merely one of multiple driving mutations and may be present in a proportion of tumor cells, whereas in epithelioid GBMs, $B R A F$ V600E mutation is likely a driving and recurrent event [7]. In the present case, FA of BRAF V600E by ddPCR was high (Additional file 2: Figure S2).

Interestingly, a recent series of 15 anaplastic PXAs reported that $100 \%$ had RAF alterations (mostly BRAF V600E and occasional BRAF or RAF1 fusions), $100 \%$ had biallelic inactivation of CDKN2A, and $47 \%$ had TERT promoter mutations [32], revealing a striking similarity of genetic alterations between anaplastic PXAs and epithelioid GBMs. Furthermore, a glioma mouse 

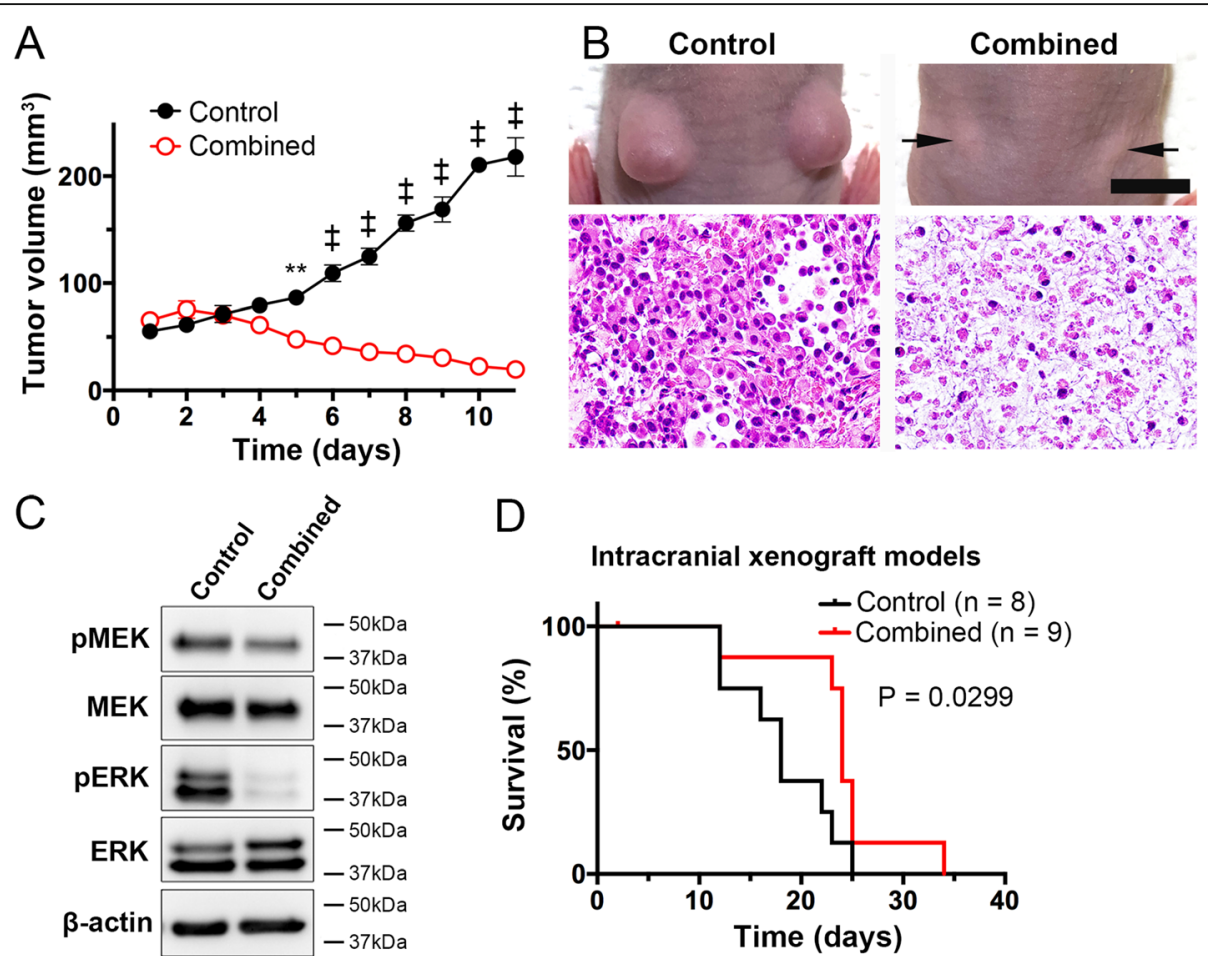

Fig. 4 Efficacy of combined treatment against subcutaneous and intracranial xenograft models. In the combined treatment group, tumor volume was significantly suppressed relative to the control group. $\left(n=4\right.$, error bars represent $\pm \operatorname{SEM}^{* * *} p=0.0021,{ }^{\ddagger} p<0.0001$; Two-way ANOVA) (a). Representative macro and micro images of the tumors from mice implanted subcutaneously with NGT41 cells (b). Western blot analysis demonstrated that expression of phosphorylated ERK was drastically inhibited by the combined treatment (c). Combined dabrafenib and trametinib treatment significantly prolonged the survival of mice in the intracranial model relative to the control $(p=0.0299$, Log Rank test) (d). Scale bars a: $10 \mathrm{~mm}$; b: $50 \mu \mathrm{m}$

model obtained by transferring the activated forms of BRAF V600E, KRAS and AKT to neural progenitor cells in Ink4a/Arf ${ }^{l o x / l o x}$ mice has been reported [33]. Deletion of Ink4a, an important tumor suppressor, produces the same effect as $C D K N 2 A$ loss (which encodes p16INK4a) in gliomas. Induction of BRAF V600E alone was not tumorigenic; Ink4a/Arf loss in combination produced well-demarcated gliomas showing evidence of growth into the subarachnoid space, recapitulating the characteristics of epithelioid GBM. Thus, the epithelioid GBM model with $B R A F$ V600E mutation in combination with $C D K N 2 A$ loss we established recapitulates the characteristics of previously published models.

Analysis of The Cancer Genome Atlas (TCGA) database included in R2: Genomics Analysis and Visualization Platform (http://r2.amc.nl) showed that $B R A F$ mutations were significantly correlated with $C D K N 2 A$ alterations $(p=0.025)$ (Additional file 2: Figure $\mathrm{S} 6 \mathrm{~A})$ and TERT promoter mutations $(p=$ 7.03e-03) (Additional file 2: Figure S6B). These three confirmed genetic alterations may be closely related to tumorigenesis in epithelioid GBM.

A recent report based on integrated molecular analysis has proposed that epithelioid GBM should be stratified into three subsets: a PXA subset with a high percentage of BRAF V600E mutations but a relatively low percentage of TERT promoter mutations, an adult $I D H$-wildtype GBM subset with a relatively low percentage of $B R A F$ V600E mutations but a high percentage of TERT promoter mutations, and a pediatric RTK1 subset not harboring either mutation [34]. This previous report illustrates the molecular and pathological complexity of epithelioid GBM, suggesting the need for cancer panel assessment before precision medicine can be considered.

To our knowledge, this is one of the first reports of epithelioid GBM characterized by leptomeningeal dissemination showing a dramatic response to combined BRAF and MEK inhibitors. Epithelioid GBMs with $B R A F$ V600E mutation showing leptomeningeal dissemination have an especially poor prognosis with a patient survival of $1-3$ months [1-3], whereas the present patient survived for 8 months after only 4 weeks of targeted therapy. Other previous reports have indicated sensitivity to BRAF inhibitor alone for gliomas with BRAF V600E mutations, the majority being gangliogliomas in children and PXAs in adults [35]. More recent reports have documented successful treatment of high-grade gliomas with $B R A F$ 
V600E mutations using a combination of dabrafenib and trametinib [35-37]. A case report illustrates stable disease for 10 months in a $B R A F$-mutant epithelioid GBM patient treated with dabrafenib alone [38]; a very recently published report shows dramatic, albeit transient response to dabrafenib and trametinib in two BRAF V600E-mutant epithelioid glioblastomas [39]. In the present case, the patient's paraplegia did not improve despite dramatic radiographical response. Findings at autopsy suggested that the tumor cells had already invaded into the spinal cord, causing irreversible destructive changes before initiation of targeted therapy.

We developed the NGT41 cell line using autopsied tumor tissue, and established a xenograft showing characteristics closely resembling those of the autopsied tumor itself, with invasion into the subarachnoid and perivascular spaces, and a mucinous stroma (Fig. 2a, b). Treatment experiments were conducted both in vitro and in vivo, and while we were able to demonstrate efficacy of the combination therapy, some of the results were not as robust as we had expected. One explanation for this is that the cell line we established was not treatment-naive. For instance, mucin was seen in the xenograft and was present in the medium after culture, whereas it was not observed at all in the initial tumor. Furthermore, it is known that additional genetic changes can occur in long-term-cultured cell lines [40]. Further genetic and epigenetic evaluation of the initial tumor, autopsy specimen, and cell line/ xenograft should be carried out in order to assess any treatment-related changes. In the patient, spinal irradiation was performed in conjunction with dabrafenib and trametinib, possibly enhancing the cytotoxicity of treatment [41], but clinical response was observed soon after starting the combined BRAF inhibitor and MEK inhibitor treatment, suggesting that targeted treatment was the main reason for the dramatic effect. Results of pMEK and pERK analysis in the intracranial tumor revealed that drug properly entered the brain (Additional file 2: Figure S5A). Also, adverse effects of the drugs were tolerable (Additional file 2: Figure S5B). Morphological findings of the HE sections suggested that tumors in the treatment group rapidly recurred after planned cessation of treatment at 14 days (Additional file 2: Figure S5C), supporting the notion that dabrafenib and trametinib should be continued until relapse. Despite these limitations, patient-derived cell lines may predict the effectiveness of targeted therapy and lead to an improved understanding of the tumor biology. Furthermore, establishment of this cell line and xenograft will potentially lead to the understanding of resistance mechanisms to BRAF and MEK inhibition and development of strategies to circumvent this resistance.

\section{Conclusions}

Dabrafenib and trametinib in combination with spinal radiation elicited a dramatic response in a patient with epithelioid GBM harboring BRAF V600E mutation, and characterized by spinal dissemination. Moreover, we established a cell line retaining the BRAF V600E mutation, which morphologically resembled the epithelioid glioblastoma, and were able to evaluate the efficacy of combined treatment. Further research using this cell line is expected to help development of new strategies to mitigate the development of resistance and augment the response to BRAF and MEK inhibition.

\section{Additional files}

\begin{abstract}
Additional file 1: Table S1. A table listing the 435 genes in the comprehensive genomic sequencing panel. (TIF $7228 \mathrm{~kb}$ )
\end{abstract}

Additional file 2: Figure S1. Genetic profiles of surgical tissue and the NGT41 cell line. BRAF V600E and TERT promotor (C250T) mutation was confirmed by Sanger sequencing $(\mathbf{A})$, and heterozygous loss of CDKN2A/2B was identified by the multiplex ligation-dependent probe amplification method (B). Figure S2. Evaluation of BRAF V600E using ddPCR. Tumor DNA was extracted from the area of vivid tumor cells in the FFPE tissue by laser microdissection (A). Fractional abundance (FA) of mutated BRAF V600E was calculated as copies of mutated DNA/(copies of mutated DNA + wildtype DNA) (B). Scale bar A: $200 \mu \mathrm{m}$. Figure S3. Calculation of growth rate value in NGT41 and U87MG after combination treatment. Dose response curves on relative cell count showed marked response to BRAF and/or MEK inhibitor treatment in NGT41 (A), but minimal reduction in U87MG (B). Figure S4. BRAF and MEK inhibitor induced greater apoptosis and G0/G1 arrest in the NGT41 cell line. In BRAF V600E-mutant cell lines, each treatment significantly increased the number of apoptotic cells $\left(n=3,{ }^{*} p<0.05,{ }^{* *} p<0.01\right.$; Two-way ANOVA) (A). G0/G1 arrest was induced by each treatment in BRAF V600E mutant-cell lines, whereas no response was observed in BRAF-wildtype cell lines $(n=3)$ (B). Figure S5. Effect of BRAF and MEK inhibitor in the intracranial model. pMEK and pERK were markedly suppressed in the treatment group (A). Serial body weight calculations in the treatment group were virtually the same as in the control group (B). Histological appearance of intracranial tumor in the treatment group at endpoint was similar to that of the control group (C). Scale bar C: $50 \mu \mathrm{m}$. Figure S6. Analysis of the TCGA database included in R2: Genomics Analysis and Visualization Platform showed that BRAF mutations were significantly correlated with CDKN2A alterations $(p=0.025)$ (A) and TERT promoter mutations ( $p=7.03 \mathrm{e}-03)$ (B). (ZIP $8066 \mathrm{~kb})$

\section{Abbreviations}

7-AAD: 7-Amino-Actinomycin D; ANOVA: analysis of variance; ATCC: American Type Culture Collection; CNS: Central nervous system; ddPCR: droplet digital polymerase chain reaction; DMEM: Dulbecco's modified Eagle medium; DMSO: Dimethyl-sulfoxide; FA: Fractional abundance; FAM: carboxyfluorescein; FBS: Fetal bovine serum; GBM: Glioblastoma; GR: Growth ratio; HE: Hematoxylin and eosin; IHC: Immunohistochemistry; IRB: Institutional review board; JCRB: Japanese Collection of Research Bioresources; MLPA: Multiplex ligation-dependent probe amplification; PI: Propidium iodide; PXA: Pleomorphic xanthoastrocytoma; TCGA: The Cancer Genome Atlas

\section{Acknowledgements}

The authors would like to acknowledge Dr. Sumihito Nobusawa of Department of Pathology, Gunma University for advice about CDKN2A2B analysis, Dr. Karisa Schreck of Department of Oncology, Johns Hopkins University, for helpful clinical advice on targeted treatment in BRAF V600E- 
mutant gliomas, and C. Tanda, J. Takasaki, Y. Tanaka, A. Nakahara, S. Nigorikawa and A. Yoshii for their technical assistance.

\begin{abstract}
Authors' contributions
YK, MOK and MNat designed the study. YK, MOk, DK, TE, JW, and SS performed experiments. AK, RS and YK performed pathological analyses. MNat, JW, YT, and MOi treated the patient. HS and HA performed radiation treatment. $\mathrm{RH}$ advised in vitro and in vivo studies. MNag and TW advised molecular analyses, TS analyzed the R2 data. YF supervised the studies. All authors read the final manuscript and made critical suggestions.
\end{abstract}

\section{Funding}

This project was supported by Denka Co., Ltd. Tokyo, Japan and in part by grants from Japan Society for Promotion of Science to MNat (17 K16632, 19 K09476), MOK (17 K17739), JW (19 K18418), YT (17 K17735) and Niigata University Brain Research Institute Global Collaborative Project 2017 between $\mathrm{RH}$ and YF. RH is supported by US National Institutes of Health grant RO1NS093079, Alex's Lemonade Stand Foundation, St. Baldrick's Foundation, Rally and Bear Necessities Pediatric Cancer Foundation, and The John McNicholas Pediatric Brain Tumor Foundation.

\section{Availability of data and materials}

The datasets used during the current study are available from the corresponding author on reasonable request. The TCGA datasets generated and/or analyzed during the current study are available in the R2 analysis and visualization platform (http://r2.amc.nl).

\section{Ethics approval and consent to participate}

The study was approved by the Institutional review board approved protocol at Niigata University (\#2016-2583). Informed consents for autopsy, collection of samples and their subsequent use for genetic analysis and other research purposes were obtained from the patient's family. IRB approval and written consent were obtained for CANCERPLEX analysis. Animal study was approved by the Animal Use and Care Committee of Niigata University.

\section{Consent for publication}

Not applicable.

\section{Competing interests}

The authors declare that they have no competing interests.

\section{Author details}

${ }^{1}$ From the Departments of Neurosurgery, Niigata University, 1-757 Asahimachidori, Chuo-ku, Niigata, Japan. ${ }^{2}$ Pathology, Brain Research Institute, Niigata University, Niigata, Japan. ${ }^{3}$ Department of Radiology and Radiation Oncology, Niigata University Graduate School of Medical and Dental Sciences, Niigata, Japan. ${ }^{4}$ Division of Digestive and General Surgery, Niigata University Graduate School of Medical and Dental Sciences, Niigata, Japan. ${ }^{5}$ Department of Neurosurgery, Northwestern University, Chicago, IL, USA.

Received: 8 June 2019 Accepted: 18 July 2019

Published online: 25 July 2019

\section{References}

1. Kuroda J, Nobusawa S, Nakamura H, Yokoo H, Ueda R, Makino K, Yano S, Kuratsu J (2016) A case of an epithelioid glioblastoma with the BRAF V600E mutation colocalized with BRAF intact low-grade diffuse astrocytoma. Neuropathology 36:181-186. https://doi.org/10.1111/neup.12258

2. Broniscer A, Tatevossian RG, Sabin ND, Klimo P, Dalton J, Lee R, Gajjar A, Ellison DW (2014) Clinical, radiological, histological and molecular characteristics of paediatric epithelioid glioblastoma. Neuropathol Appl Neurobiol 40:327-336. https://doi.org/10.1111/nan.12093

3. He MX, Wang JJ (2011) Rhabdoid glioblastoma: case report and literature review. Neuropathology 31:421-426. https://doi.org/10.1111/ j.1440-1789.2010.01166.x

4. Kleinschmidt-DeMaster BK, Alassiri AH, Birks DK, Newell KL, Moore W, Lillehei KO (2010) Epithelioid versus rhabdoid glioblastomas are distinguished by monosomy 22 and immunohistochemical expression of INI-1 but not claudin 6. Am J Surg Pathol 34:341-354. https://doi. org/10.1097/PAS.0b013e3181ce107b
5. Momota H, Iwami K, Fujii M, Motomura K, Natsume A, Ogino J, Hasegawa T, Wakabayashi T (2011) Rhabdoid glioblastoma in a childcase report and literature review. Brain Tumor Pathol 28:65-70. https:// doi.org/10.1007/s10014-010-0010-4

6. Louis DN, Ohgaki H, Wiestler OD, Cavenee WK, Ellison DW, Figarella-Branger D, Perry A, Reifenberger G, von Deimling A (2016) WHO classification of tumours of the central nervous system. IARC, Lyon

7. Nakajima N, Nobusawa S, Nakata S, Nakada M, Yamazaki T, Matsumura N, Harada K, Matsuda H, Funata N, Nagai S, Nakamura H, Sasaki A, Akimoto J, Hirato J, Yokoo H (2018) BRAF V600E, TERT promoter mutations and CDKN2A/B homozygous deletions are frequent in epithelioid glioblastomas: a histological and molecular analysis focusing on intratumoral heterogeneity. Brain Pathol 28:663-673. https://doi.org/1 $0.1111 /$ bpa. 12572

8. Kleinschmidt-DeMasters BK, Aisner DL, Birks DK, Foreman NK (2013) Epithelioid GBMs show a high percentage of BRAF V600E mutation. Am J Surg Pathol 37:685-698. https://doi.org/10.1097/PAS.0b013e31827f9c5e

9. Maemondo M, Inoue A, Kobayashi K, Sugawara S, Oiszumi S, Isobe H, Yoshizawa H, Kinoshita I, Fujita Y, Okinaga S (2010) Gefitinib or chemotherapy for non-small-cell lung cancer with mutated EGFR. N Engl J Med 362:2380-2388. https://doi.org/10.1056/NEJMoa0909530

10. Baselga J, Cortes J, Kim S, Im S, Hegg R, Im Y, Roman L, Pedrini JL, Pienkowski T, Knott A, Clark EA, Benyunes MC, Ross G, Swain SM (2012) Pertuzumab plus trastuzumab plus docetaxel for metastatic breast cancer. N Engl J Med 366:109-119. https://doi.org/10.1056/NEJMoa1113216

11. del Bufalo F, Carai A, Fig-Talamanca L, Pettorini B, Malluci C, Giangaspero F, Antonelli M, Badiali M, Moi L, Bianco G, Cacchione A, Locatelli F, Ferretti E, Mastronuzzi A (2014) Response of recurrent BRAFV600E mutated ganglioglioma to Vemurafenib as single agent. J Transl Med 12:356. https:// doi.org/10.1186/s12967-014-0356-1

12. Bautista F, Paci A, Minard-Colin V, Dufour C, Grill J, Lacroix L, Varlet P, Valteau-Couanet D, Geoerger B (2014) Vemurafenib in pediatric patients with BRAFV600E mutated high-grade gliomas. Pediatr Blood Cancer 61: 1101-1103. https://doi.org/10.1002/pbc.24891

13. Brown NF, Carter T, Kitchen N, Mulholland P (2017) Dabrafenib and trametinib in BRAFV600E mutated glioma. CNS Oncol 6:291-296

14. Lee EQ, Ruland S, LeBoeuf NR, Wen PY, Santagata S (2010) Successful treatment of a progressive BRAF V600E-mutated anaplastic pleomorphic xanthoastrocytoma with vemurafenib monotherapy. J Clin Oncol 34:e87e89. https://doi.org/10.1200/JCO.2013.51.1766

15. Brastianos PK, Shankar GM, Gill CM, Taylor-Weiner A, Nayyar N, Panka DJ, Sullivan RJ, Frederick DT, Abedalthagafi M, Jones PS, Dunn IF, Nahed BV, Romero JM, Louis DN, Getz G, Cahill DP, Santagata S, Curry WT, Jr., Barker FG, 2nd (2016) Dramatic response of BRAF V600E mutant papillary Craniopharyngioma to targeted therapy. J Natl Cancer Inst 108https://doi. org/10.1093/jnci/djv310

16. Aylwin SJ, Bodi I, Beaney R (2016) Pronounced response of papillary craniopharyngioma to treatment with vemurafenib, a BRAF inhibitor. Pituitary 19:544-546. https://doi.org/10.1007/s11102-015-0663-4

17. Kaley T, Touat M, Subbiah V, Hollebecque A, Rondon J, Lockhart C, Keedy V, Bielle F, Hofheinz R, Joly F, Blay J, Chau I, Puzanov I, Raje NS, Wolf J, DeAngelis LM, Makrutzki M, RiehI T, Pitcher B, Baselga J, Hyman DM (2018) BRAF inhibition in BRAFV600-mutant gliomas- results from the VE-BASKET study. J Clin Oncol 36:3477-3484. https://doi.org/10.1200/JCO.2018

18. Natsumeda M, Aoki H, Miyahara H, Yajima N, Uzuka T, Toyoshima Y, Kakita A, Takahashi H, Fujii Y (2011) Induction of autophagy in temozolomide treated malignant gliomas. Neuropathology 31:486493. https://doi.org/10.1111/j.1440-1789.2010.01197.x

19. Ogura R, Tsukamoto $Y$, Natsumeda M, Isogawa M, Aoki H, Kobayashi T, Yoshida S, Okamoto K, Takahashi H, Fujii Y, Kakita A (2015) Immunohistochemical profiles of IDH1, MGMT and P53: practical significance for prognostication of patients with diffuse gliomas. Neuropathology 35:324-335. https://doi.org/10.1111/neup.12196

20. Ichikawa H, Nagahashi M, Shimada Y, Hanyu T, Ishikawa T, Kameyama H, Kobayashi T, Sakata J, Yabusaki H, Nakagawa S, Sato N, Hirata Y, Kitagawa Y, Tanahashi T, Yoshida K, Nakanishi R, Oki E, Vuzman D, Lyle S, Takabe K, Ling Y, Okuda S, Akazawa K, Wakai T (2017) Actionable gene-based classification toward precision medicine in gastric cancer. Genome Med 9:93. https://doi.org/10.1186/s13073-017-0484-3

21. Watanabe J, Natsumeda M, Okada M, Kobayashi D, Kanemaru Y, Tsukamoto Y, Oishi M, Kakita A, Fujii Y (2019) High detection rate of 
MYD88 mutations in cerebrospinal fluid from patients with central nervous system lymphomas. JCO Precis OncolDOI. https://doi.org/1 0.1200/PO.18.00308

22. Jeuken J, Sijben A, Alenda C, Rijntjes J, Dekkers M, Boots-Sprenger S, McLendon R, Wesseling P (2009) Robust detection of EGFR copy number changes and EGFR variant III: technical aspects and relevance for glioma diagnostics. Brain Pathol 19:661-671. https://doi.org/10.1111/ j.1750-3639.2009.00320.x

23. Hafner M, Niepel M, Chung M, Sorger PK (2016) Growth rate inhibition metrics correct for confounders in measuring sensitivity to cancer drugs. Nat Methods 13:521-527. https://doi.org/10.1038/nmeth.3853

24. Natsumeda M, Maitani K, Liu Y, Miyahara H, Kaur H, Chu Q, Zhang H, Kahlert UD, Eberhart CG (2016) Targeting notch signaling and autophagy increases cytotoxity in glioblastoma neurospheres. Brain Pathol 26:254-262. https:// doi.org/10.1111/bpa.12343

25. Kawasaki A, Okada M, Tamada A, Okuda S, Nozumi M, Ito Y, Kobayashi D, Yamasaki T, Yokoyama R, Shibata T, Nishina H, Yoshida Y, Fujii Y, Takeuchi K, Igarashi M (2018) Growth cone phosphoproteomics reveals that GAP-43 phosphorylated by JNK is a marker of axon growth and regeneration. iScience 4:190-203. https://doi.org/10.1016/j.isci.2018.05.019

26. Tsukamoto $Y$, Ohtsu N, Echizenya S, Otsuguro S, Ogura R, Natsumeda M, Isogawa M, Aoki H, Ichikawa S, Sakaitani M, Matsuda A, Maenaka K, Fujii Y, Kondo T (2016) Chemical screening identifies EUrd as a novel inhibitor against temozolomide-resistant glioblastoma-initiating cells. Stem Cells 34 2016-2025. https://doi.org/10.1002/stem.2380

27. Glassmann A, Reichmann K, Scheffler B, Glas M, Veit N, Probstmeier R (2011) Pharmacological targeting of the constitutively activated MEK/MAPKdependent signaling pathway in glioma cells inhibits cell proliferation and migration. Int J Oncol 39:1567-1575. https://doi.org/10.3892/ijo.2011.1165

28. Robert C, Karaszewska B, Schachter J, Rutkowski P, Mackiewicz A, Stroiakovski D, Lichinitser M, Dummer R, Grange F, Mortier L, Chiarion-Sileni V, Drucis K, Krajsova I, Hauschild A, Lorigan P, Wolter P, Long GV, Flaherty K, Nathan P, Ribas A, Martin AM, Sun P, Crist W, Legos J, Rubin SD, Little SM, Schadendorf D (2015) Improved overall survival in melanoma with combined dabrafenib and trametinib. N Engl J Med 372:30-39. https://doi. org/10.1056/NEJMoa1412690

29. Long GV, Stroyakovskiy D, Gogas H, Levchenko E, de Braud F, Larkin J, Garbe C, Jouary T, Hauschild A, Grob J-J, Chiarion-Sileni V, Lebbe C, Mandalà M, Millward M, Arance A, Bondarenko I, Haanen JBAG, Hansson J, Utikal J, Ferraresi V, Kovalenko N, Mohr P, Probachai V, Schadendorf D, Nathan P, Robert C, Ribas A, DeMarini DJ, Irani JG, Swann S, Legos JJ, Jin F, Mookerjee B, Flaherty K (2015) Dabrafenib and trametinib versus dabrafenib and placebo for Val600 BRAF-mutant melanoma: a multicentre, double-blind, phase 3 randomised controlled trial. Lancet 386:444-451. https://doi.org/1 0.1016/s0140-6736(15)60898-4

30. Nazarian R, Shi H, Wang Q, Kong X, Koya RC, Lee $H$, Chen Z, Lee M-K, Attar N, Sazegar H, Chodon T, Nelson SF, McArthur G, Sosman JA, Ribas A, Lo RS (2010) Melanomas acquire resistance to B-RAF(V600E) inhibition by RTK or N-RAS upregulation. Nature 468:973-977. https:// doi.org/10.1038/nature09626

31. Zhang J, Yao T-W, Hashizume R, Hariono S, Barkovich KJ, Fan Q-W, Prados M, James CD, Weiss WA, Nicolaides T (2016) Combined BRAFV600E and MEK blockade for BRAFV600E-mutant gliomas. J Neuro-Oncol 131:495-505. https://doi.org/10.1007/s11060-016-2333-4

32. Phillips JJ, Gong H, Chen K, Joseph NM, van Ziffle J, Bastian BC, Grenert JP, Kline CN, Mueller S, Banerjee A, Nicolaides T, Gupta N, Berger MS, Lee HS, Pekmezci M, Tihan T, Bollen AW, Perry A, Shieh JTC, Solomon DA (2019) The genetic landscape of anaplastic pleomorphic xanthoastrocytoma. Brain Pathol 29:85-96. https://doi.org/10.1111/bpa.12639

33. Robinson JP, VanBrocklin MW, Guilbeault AR, Signorelli DL, Brandner $S$, Holmen SL (2010) Activated BRAF induces gliomas in mice when combined with Ink4a/Arf loss or Akt activation. Oncogene 29:335-344. https://doi. org/10.1038/onc.2009.333

34. Korshunov A, Chavez L, Sharma T, Ryzhova M, Schrimpf D, Stichel D, Capper D, Sturm D, Kool M, Habel A, Kleinschmidt-DeMasters BK, Rosenblum M, Absalyamova O, Golanov A, Lichter P, Pfister SM, Jones DTW, Perry A, von Deimling A (2018) Epithelioid glioblastomas stratify into established diagnostic subsets upon integrated molecular analysis. Brain Pathol 28:656662. https://doi.org/10.1111/bpa.12566

35. Johanns TM, Ferguson CJ, Grierson PM, Dahiya S, Ansstas G (2018) Rapid clinical and radiographic response with combined dabrafenib and trametinib in adults with BRAF-mutated high-grade glioma. J Natl Compr Cancer Netw 16:4-10. https://doi.org/10.6004/jnccn.2017.7032

36. Toll SA, TH N, Cotter J, Judkins AR, Tamrazi B, Biegerl JA, Dhall G, Robinson NJ, Waters K, Patel P, Cooper R, Margol AS (2019) Sustained response of three pediatric BRAFV600E mutated high-grade gliomas to combined BRAF and MEK inhibitor therapy. Oncotarget 10:551-557. https://doi.org/10.18632/oncotarget.26560

37. Schreck KC, Guajardo A, Lin DDM, Eberhart CG, Grossman SA (2018) Concurrent BRAF/MEK inhibitors in BRAF V600-mutant high-grade primary brain tumors. J Natl Compr Cancer Netw 16:343-347. https:// doi.org/10.6004/jncen.2017.7052

38. Ceccon G, Werner JM, Dunkl V, Tscherpel C, Stoffels G, Brunn A, Deckert M, Fink GR, Galldiks N (2018) Dabrafenib treatment in a patient with an epithelioid glioblastoma and BRAF V600E mutation. Int J Mol Sci 19https:// doi.org/10.3390/ijms19041090

39. Woo PYM, Lam T, PJK S, Li L, Leung RCY, Ho JMK, Zhung JTF, Wong B, Chan TSK, Loong HHF, Ng HK (2019) Regression of BRAFV600E mutant adult glioblastoma after primary combined BRAF-MEK inhibitor targeted therapy: a report of two cases. Oncotarget 10:3818-3826

40. Li A, Walling J, Kotliarov Y, Center A, Steed ME, Ahn SJ, Rosenblum M, Mikkelsen T, Zenklusen JC, Fine HA (2008) Genomic changes and gene expression profiles reveal that established glioma cell lines are poorly representative of primary human gliomas. Mol Cancer Res 6:21-30. https:// doi.org/10.1158/1541-7786.MCR-07-0280

41. Dasgupta T, Olow AK, Yang X, Hashizume R, Nicolaides TP, Tom M, Aoki Y, Berger MS, Weiss WA, Stalpers LJ, Prados M, James CD, Mueller S, HaasKogan DA (2016) Survival advantage combining a BRAF inhibitor and radiation in BRAF V600E-mutant glioma. J Neuro-Oncol 126:385-393. https:// doi.org/10.1007/s11060-015-1939-2

\section{Publisher's Note}

Springer Nature remains neutral with regard to jurisdictional claims in published maps and institutional affiliations.

\section{Ready to submit your research? Choose BMC and benefit from:}

- fast, convenient online submission

- thorough peer review by experienced researchers in your field

- rapid publication on acceptance

- support for research data, including large and complex data types

- gold Open Access which fosters wider collaboration and increased citations

- maximum visibility for your research: over $100 \mathrm{M}$ website views per year

At BMC, research is always in progress.

Learn more biomedcentral.com/submissions 